\title{
Coherent phonon manipulation in coupled mechanical resonators
}

\author{
Hajime Okamoto ${ }^{1 \star}$, Adrien Gourgout ${ }^{1}$, Chia-Yuan Chang ${ }^{1,2}$, Koji Onomitsu ${ }^{1}$, Imran Mahboob ${ }^{1}$, \\ Edward Yi Chang ${ }^{2}$ and Hiroshi Yamaguchi ${ }^{1}$
}

\begin{abstract}
Coupled nanomechanical resonators have recently attracted great attention for both practical applications and fundamental studies owing to their sensitive sympathetic oscillation dynamics ${ }^{1-10}$. A challenge to the further development of this architecture is the coherent manipulation of the coupled oscillations. Here, we demonstrate strong dynamic coupling between two GaAs-based mechanical resonators by periodically modulating (pumping) the stress using a piezoelectric transducer. This strong coupling enables coherent transfer of phonon populations between the resonators, namely phonon Rabi oscillations ${ }^{11-13}$. The nature of the dynamic coupling can also be tuned from a linear first-order interaction to a nonlinear higher-order process in which more than one pump phonon mediates the coherent oscillations (that is, multi-pump phonon mixing). This coherent manipulation is not only useful for controlling classical oscillations ${ }^{14}$ but can also be extended to the quantum regime ${ }^{11-13}$, opening up the prospect of entangling two distinct macroscopic mechanical objects ${ }^{15,16}$.
\end{abstract}

The mechanical motion of two harmonic oscillators can interact if the oscillators are geometrically interconnected. Such coupled oscillations were first observed in paired pendulum clocks in the mid-seventeenth century and were extensively studied for their novel sympathetic oscillation dynamics ${ }^{17,18}$. In this era of nanotechnologies, coupled oscillations have again emerged as subjects of interest when realized in nanomechanical resonators owing to their potential applications in highly precise sensors ${ }^{1-3}$, high-quality-factor band-pass filters ${ }^{4}$, signal amplifiers ${ }^{5}$ and logic gates ${ }^{6}$. However, a key obstacle to the further development of this platform is the ability to coherently manipulate the coupling between different mechanical oscillations. This limitation arises as a consequence of the usually weak vibration coupling between the constituent nanomechanical elements. In this Letter, we demonstrate dynamic coupling between two geometrically interconnected GaAs doubly-clamped beams by using piezoelectrically induced parametric mode mixing (pumping) ${ }^{19,20}$. This technique enables coherent manipulation of phonon populations as well as strong vibration coupling in frequency-different mechanical resonators in which the energy exchange between the two resonators is intrinsically inefficient owing to the frequency mismatch.

The dynamic coupling is developed in paired GaAs-based mechanical beams, as shown in Fig. 1a, in which the piezoelectric effect is exploited to mediate all-electrical displacement transduction ${ }^{19,20}$. Application of the gate voltage causes piezoelectric stress along the beam, resulting in modulation of its tension and the creation of a bending moment. This effect enables both harmonic driving and parametric pumping, where the mechanical motion can be detected by the voltage induced by the piezoelectric effect. All the measurements were done by setting the sample in a vacuum $\left(5 \times 10^{-5} \mathrm{~Pa}\right)$ and cooling it to $1.5 \mathrm{~K}$ with a ${ }^{4} \mathrm{He}$ cryostat.

The frequency response of beam 1 measured by harmonically driving it while the parametric pump is deactivated exhibits two coupled vibration modes (Fig. 1b), where mode 1 $\left(\omega_{1}=2 \pi \times 293.93 \mathrm{kHz}\right)$ is dominated by the vibration of beam 1 while mode $2\left(\omega_{2}=2 \pi \times 294.37 \mathrm{kHz}\right)$ is dominated by the vibration of beam 2 . The amplitude of mode 2 is much smaller than that of mode 1, reflecting the energy exchange due to the structural coupling via the overhang is inefficient because of the eigenfrequency difference between the two beams. The quality factor of these modes is $Q \sim 14,000$ with a frequency- $Q$ product of $4 \times 10^{9}$ (ref. 21). The major contribution to the energy dissipation $(1 / Q)$ arises from the clamping losses ${ }^{22}$, while the thermoelastic dissipation is negligible at cryogenic temperatures ${ }^{23}$.

The frequency difference between the two modes can be compensated by activating the parametric pump, which results in mixing between the two vibration modes. This is induced by piezoelectrically modulating the spring constant of beam 1 with the pump frequency $\omega_{\mathrm{p}}$ at around the frequency difference between the two modes, $\Delta \omega \equiv \omega_{2}-\omega_{1}$ (Fig. 1c). The resulting dynamics can then be expressed by the following equations of motion:

$$
\ddot{x}_{1}+\gamma_{1} \dot{x}_{1}+\left[\omega_{1}^{2}+\Gamma_{1} \cos \left(\omega_{\mathrm{p}} t\right)\right] x_{1}+\Lambda \cos \left(\omega_{\mathrm{p}} t\right) x_{2}=F_{1} \cos \left(\omega_{d} t+\phi\right)
$$

$\ddot{x}_{2}+\gamma_{2} \dot{x}_{2}+\left[\omega_{2}^{2}+\Gamma_{2} \cos \left(\omega_{\mathrm{p}} t\right)\right] x_{2}+\Lambda \cos \left(\omega_{\mathrm{p}} t\right) x_{1}=F_{2} \cos \left(\omega_{d} t+\phi\right)$,

where $x_{i}(i=1,2)$ is the displacement of the $i$-th mode, $\omega_{i}$ is the mode frequency, $\gamma_{i}\left(=\omega_{i} / Q_{i}\right)$ is the energy dissipation rate, $F_{i}$ is the drive force $\left(F_{1} \gg F_{2}\right), \omega_{d}$ is the drive frequency and $\Gamma_{i}$ and $\Lambda$ are the intra- and inter-modal coupling coefficients respectively. When the frequency mismatch between mode 1 and mode 2 is compensated by activating the pump at $\omega_{\mathrm{p}} \simeq \Delta \omega$, the terms containing $\Lambda$ transfer phonons (oscillations) from one mode to the other (Fig. 1d). This inter-modal coupling can also be explained by the mixing of mode 1 (2) and the Stokes sideband, $\omega_{2}-\omega_{\mathrm{p}}$ (the anti-Stokes sideband, $\omega_{1}+\omega_{\mathrm{p}}$ ) of mode 2 (1) leading to normalmode splitting in the strong-coupling regime ${ }^{19,24-26}$ (Fig. 1d). The equations also include terms proportional to $\Gamma_{i}$. These terms lead to intra-modal coupling, which becomes significant for the higher-order couplings shown later. The above model can

${ }^{1}$ NTT Basic Research Laboratories, Nippon Telegraph and Telephone Corporation, Atsugi 243-0198, Japan, ${ }^{2}$ The Department of Materials Science and Engineering, National Chiao-Tung University, Hsinchu 980-8578, China. ${ }^{\star}$ e-mail: okamoto.hajime@lab.ntt.co.jp 
a

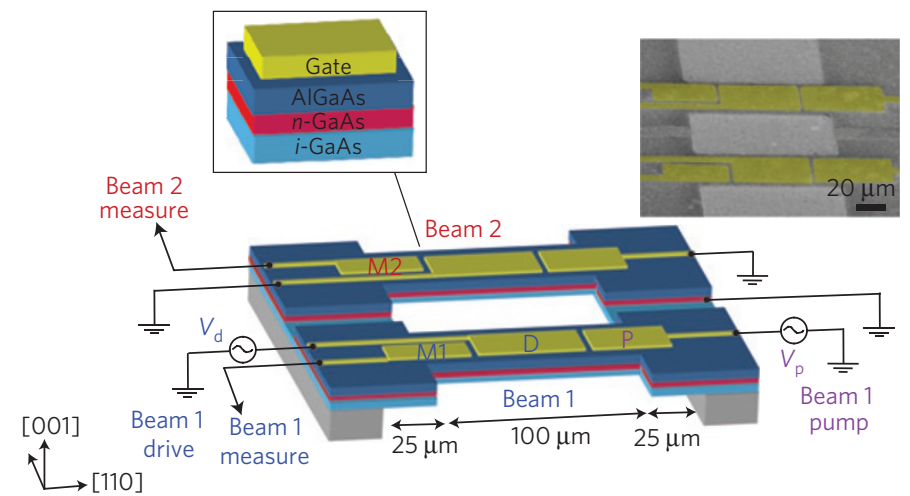

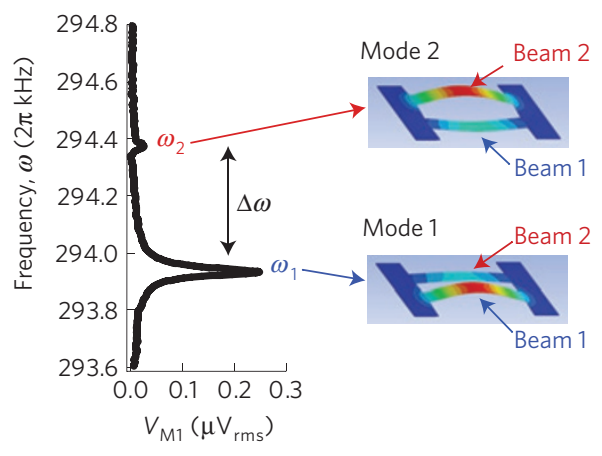

c

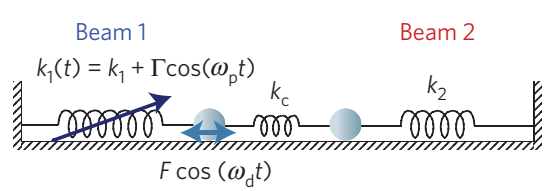

d

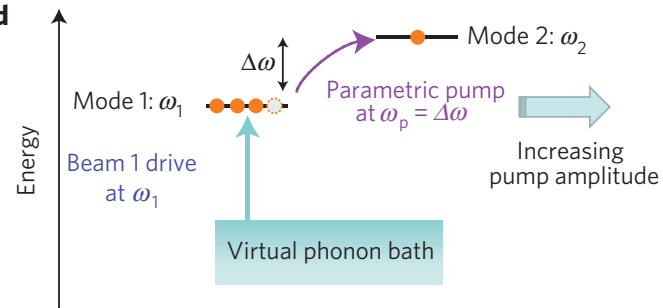

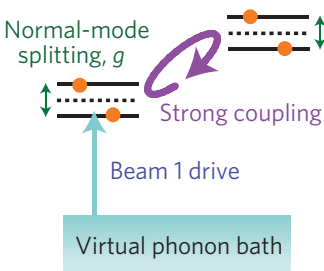

Figure 1 | Paired mechanical resonators and the parametric pumping scheme. a, Schematic of the sample, the measurement and a false-colour scanning electron micrograph. The two doubly clamped beams are structurally interconnected by the $25-\mu \mathrm{m}$ coupling overhang. b. Frequency response of beam 1 measured at gate $\mathrm{M} 1$ by driving it with $V_{d}=1.5 \mathrm{mV} V_{p-p}$ at gate $\mathrm{D}$ while the pump is deactivated $\left(V_{p}=0 V_{p-p}\right)$. The mode shapes at $\omega_{1}$ and $\omega_{2}$ obtained by finite element method calculations are also shown (exaggerated for clarity). c, Schematic of the parametric pumping protocol in an equivalent spring model. $k_{1}, k_{2}$, and $k_{c}$ are respectively the spring constant of beam 1 , beam 2 , and the coupling overhang. $\Gamma$ represents the parametric pump amplitude. $\mathbf{d}$, Schematic of the parametric pumping protocol in an energy diagram.

reproduce all the experimental results and is described in detail in Supplementary Information.

The normal-mode splitting for modes 1 and 2 was experimentally confirmed by applying a pump with voltage $V_{\mathrm{p}}$ at $\omega_{\mathrm{p}}$ in addition to the weak harmonic driving at $\omega_{d}$ to beam 1 (Fig. 1a). Probing the modes via beam 1 while the parametric pump is activated shows that mode 1 splits into two when $\omega_{\mathrm{p}} \simeq \Delta \omega=2 \pi \times 0.44 \mathrm{kHz}$, clearly demonstrating strong coupling (Fig. $2 \mathrm{a}-\mathrm{c}$ ). The transfer of phonons from mode 1 to mode 2 can also be confirmed in the response of beam 2 which was detected at frequency $\omega_{d}+\omega_{\mathrm{p}}$ (Fig. $2 \mathrm{~d}-\mathrm{f}$ ). This measurement indicates the creation of a vibration in mode 2 that is excited by the parametric pump. A phonon reaction picture can help to understand this elementary process, where phonons are created in mode 2 at the expense of probe phonons in mode 1 and pump phonons, that is, via the one-pump phonon absorption process, $\hbar \omega_{1}+\hbar \omega_{\mathrm{p}} \rightarrow \hbar \omega_{2}$ (Figs 1d and $3 \mathrm{~h}$ ). The strong coupling in the large pump regime also results in the reverse emission process, $\hbar \omega_{2} \rightarrow \hbar \omega_{1}+\hbar \omega_{\mathrm{p}}$ (Fig. 1d).

The $V_{\mathrm{p}}$ dependence of the mode splitting at $\omega_{\mathrm{p}}=\Delta \omega$ shows that the coupling strength is highly controllable (Fig. 2i). The linear $V_{\mathrm{p}}$ dependence is due to the fact that the inter-modal coupling coefficient, $\Lambda$, is proportional to $V_{\mathrm{p}}$, which can be theoretically reproduced by equations (1a) and (1b) (Fig. 2k). The separation between the split peaks provides the coupling rate, $g$, which can become so large that it can exceed the intrinsic energy dissipation rate of the two modes $\left(\gamma_{1} \simeq \gamma_{2}=2 \pi \times 21 \mathrm{~Hz}\right)$ by more than a factor of four $\left(g=2 \pi \times 90 \mathrm{~Hz}\right.$ for $\left.V_{\mathrm{p}}=1.0 \mathrm{~V}_{\mathrm{p}-\mathrm{p}}\right)$.

More remarkably, additional mode splittings in which the pump frequency does not correspond to the frequency difference between the two modes can also be observed. For example, the splitting occurs when $\omega_{\mathrm{p}} \simeq \Delta \omega / 2=2 \pi \times 0.22 \mathrm{kHz}$ for both modes 1 and 2 (Fig. 2c). This splitting is caused by a second-order coupling via a two-pump phonon absorption/emission process, that is, $\hbar \omega_{1}+2 \hbar \omega_{\mathrm{p}} \leftrightarrow \hbar \omega_{2}$, which leads to the coupling between mode
1 (2) and the second Stokes sideband, $\omega_{2}-2 \omega_{\mathrm{p}}$ (the second anti-Stokes sideband, $\omega_{1}+2 \omega_{\mathrm{p}}$ ) of mode $2(1)$. The $V_{\mathrm{p}}$ dependence of this mode splitting indicates that it has a parabolic dependence (Fig. 2j). This is because the second-order process requires a two-step phonon excitation path from mode 1 to mode 2 , and vice versa, through the intermediary energy level $\left(\omega_{1}+\omega_{2}\right) / 2$ via both the intra-modal coupling $\left(\Gamma_{i} \propto V_{\mathrm{p}}\right)$ and the inter-modal coupling $\left(\Lambda \propto V_{\mathrm{p}}\right)$, therefore, $\left[\Gamma_{i} \times \Lambda\right] \propto V_{\mathrm{p}}^{2}$, as shown in Fig. $3 i$. The corresponding mode splitting shows good agreement with the theoretical simulations (Fig. 2l). Consequently, the additional mode splittings observed in Fig. 2c correspond to even higher order coupling processes, requiring more than two pump phonons.

The strong dynamic coupling between the two mechanical resonators opens up a path to coherent control of the coupled mechanical oscillations. The time-domain measurements using the pulse sequence shown in Fig. 3a enable us to observe coherent and periodic energy exchange between the two beams/modes. The pump frequency dependence of the time-domain response of beam 2 at $\omega_{2}$ clearly shows the periodic amplitude oscillations at $\omega_{\mathrm{p}} \simeq \Delta \omega$ for the first-order $(n=1)$ coupling (Fig. 3b), which corresponds to a one-step phonon process (Fig. $3 \mathrm{~h}$ ). The $V_{\mathrm{p}}$ dependence at $\omega_{\mathrm{p}}=\Delta \omega$ shows that the vibration energy of mode 1 (beam 1) can be transferred to mode 2 (beam 2) and back eight times before energy relaxation at $V_{\mathrm{p}}=1.0 \mathrm{~V}_{\mathrm{p}-\mathrm{p}}$ (Fig. 3d). Coherent energy exchange for the second-order $(n=2)$ coupling can also be observed at $\omega_{\mathrm{p}} \simeq \Delta \omega / 2$ (Fig. 3b), where up to five oscillation periods are observed in the range of $V_{\mathrm{p}} \leq 1.0 \mathrm{~V}_{\mathrm{p}-\mathrm{p}}$ (Fig. 3e). The coupling rate, $g$, extracted from the Fourier transforms of the time-domain response, is proportional to $V_{\mathrm{p}}$ for the first-order coupling and $V_{\mathrm{p}}^{2}$ for the second-order coupling (Fig. 4). These coupling rates correspond perfectly to the mode splitting observed in the frequency response measurements.

The time-domain measurements also enable us to observe higher-order coupling, that is, $n \geq 3$. Figure $3 \mathrm{f}, \mathrm{g}$ show 
Experiment

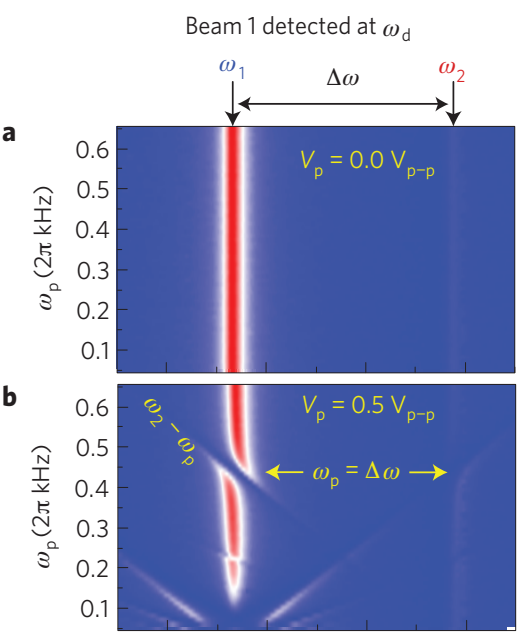

c
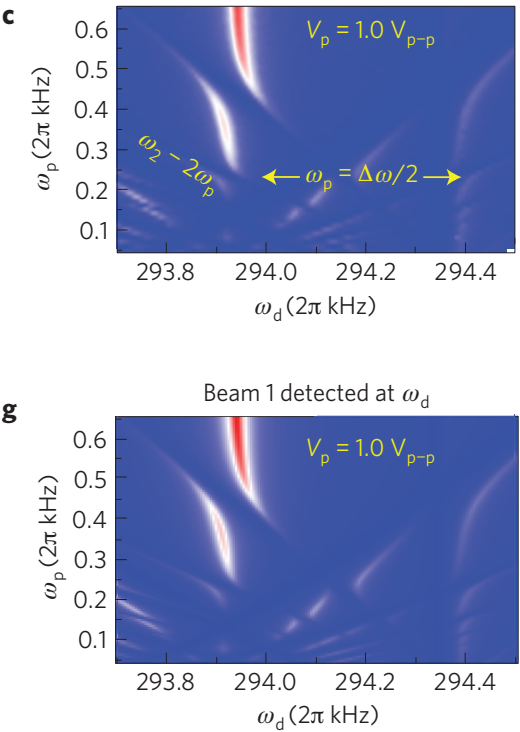

d

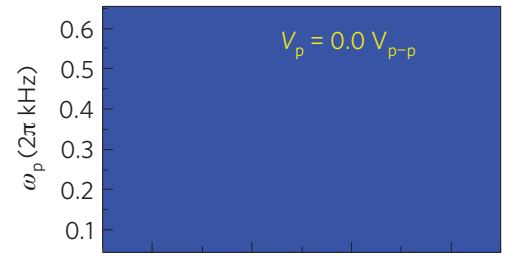

e

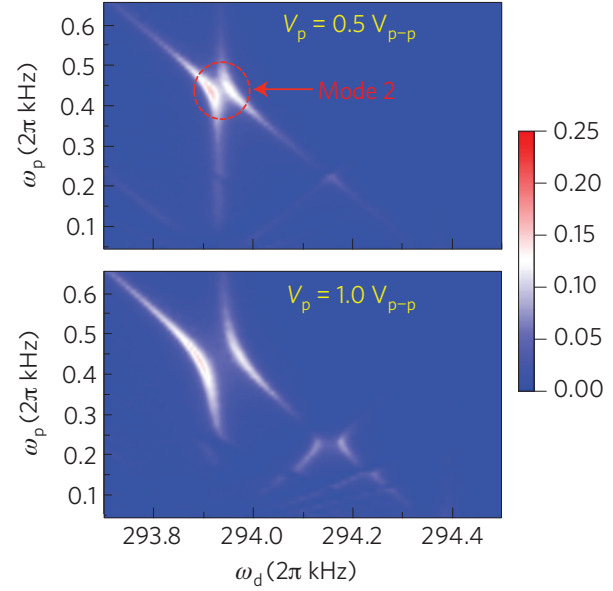

Simulation

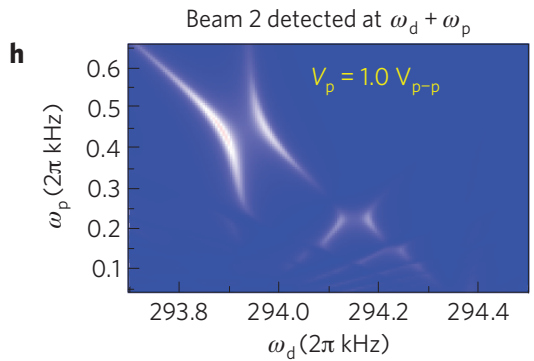

Experiment

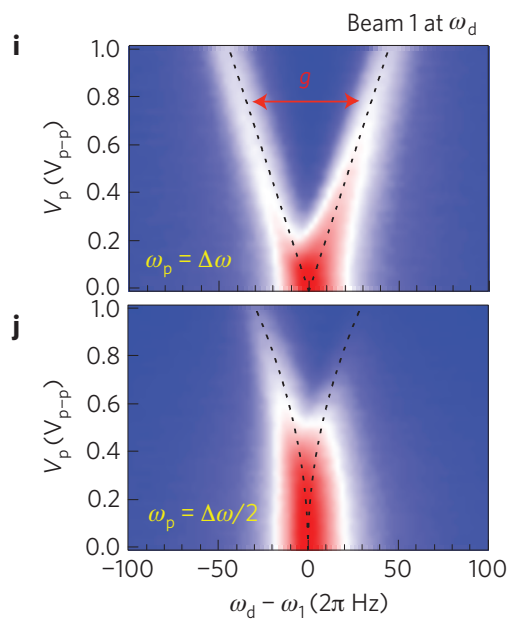

Simulation

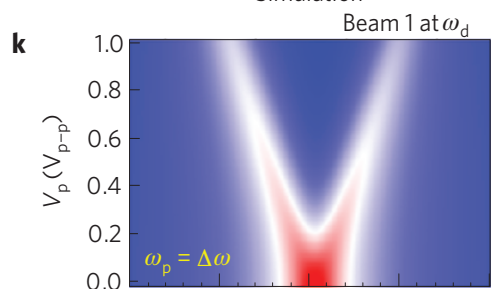

I

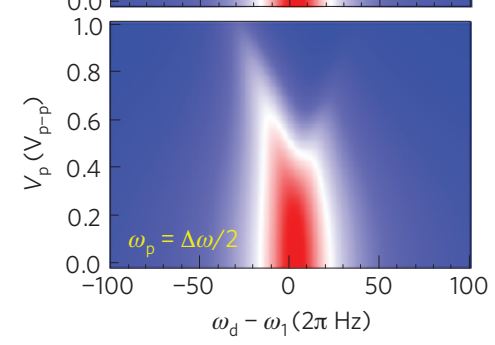

Figure 2 | Dynamic mode coupling induced by parametric pumping. a-c, The drive frequency $\left(\omega_{d}\right)$ and the pump frequency $\left(\omega_{p}\right)$ response of beam 1 detected at frequency $\omega_{d}$ for three different pump voltages, $V_{p}=0,0.5$ and $1.0 V_{p-p}$. In these measurements, beam 1 was driven by a continuous a.c. voltage $\left(V_{d}=1.5 \mathrm{mV} V_{p-p}\right)$ applied to gate $D$ while the frequency, $\omega_{d}$, was swept around the two modes. A continuous a.c. pump voltage $\left(V_{p}\right)$ was simultaneously applied to gate $\mathrm{P}$ while its frequency, $\omega_{\mathrm{p}}$, was also swept. The response of beam 1 at frequency $\omega_{d}$ was monitored via gate M1 with a pre-amplifier and a lock-in detector (see Supplementary Information). d-f, The $\omega_{d}$ and $\omega_{\mathrm{p}}$ response of beam 2 measured via gate M2 at frequency $\omega_{d}+\omega_{\mathrm{p}}$ for $V_{p}=0,0.5$ and $1.0 V_{p-p} . \mathbf{g}, \mathbf{h}$, Simulation results for $\mathbf{c}$ and $\mathbf{f}$, which were performed for the theoretical model expressed by equations (1a) and (1b). $\mathbf{i}, \mathbf{j}$, The $V_{p}$ dependence of the splitting of mode 1 induced by the first- and second-order coupling at $\omega_{p}=\Delta \omega$ and $\Delta \omega / 2$, respectively. The broken curves in $\mathbf{i}, \mathbf{j}$ represent the theoretical values of the mode splitting, which are proportional to $V_{p}$ and $V_{p}^{2}$ respectively. The mode splitting corresponds to the coupling rate, $g . \mathbf{k}, \mathbf{l}$, Simulation results for the splitting of mode 1 for $\omega_{p}=\Delta \omega$ and $\Delta \omega / 2$, respectively. Colour scale applies to all figure parts.

the coherent energy exchange between the two modes for $\omega_{\mathrm{p}}=\Delta \omega / 3=2 \pi \times 0.147 \mathrm{kHz}$ and $\omega_{\mathrm{p}}=\Delta \omega / 4=2 \pi \times 0.11 \mathrm{kHz}$, respectively. These coherent oscillations are caused by the $n$-pump phonon absorption/emission processes, that is, $\hbar \omega_{1}+n \hbar \omega_{\mathrm{p}} \leftrightarrow \hbar \omega_{2}$, through the intermediary energy levels via intra- and inter-modal coupling, for example, $\left[\Gamma_{1} \times \Gamma_{1} \times \Lambda\right]$ and $\left[\Gamma_{1} \times \Gamma_{1} \times \Gamma_{1} \times \Lambda\right]$, as shown in Fig. 3j,k. The Fourier transforms of the time-domain response reveal that the coupling rate exhibits a $V_{\mathrm{p}}^{n}$ dependence even for $n \geq 3$ (Fig. 4), which again shows good agreement with the theoretical model.

The present results show that electromagnetic pulse techniques, which are commonly used to coherently manipulate quantum two-level systems ${ }^{11,27}$, can also be applied to coherently control mechanical systems. By tuning the parametric-pump frequency, multi-wave phonon mixing involving an arbitrary number of pump phonons can be achieved in an analogous fashion to multi-wave photon mixing ${ }^{28}$. The parametric pumping allows highly controllable time-domain manipulation of phonon populations in the two modes simply by the adjustment of the pumppulse duration, thus permitting $\pi$ and $\pi / 2$-pulse operations on the Bloch sphere ${ }^{11}$ (see also independent experiments at LMU with a single mechanical resonator ${ }^{29}$ ). This coherent control further expands the applications of mechanical resonators, including the high-speed operation of high- $Q$ mechanical resonators ${ }^{14}$ and mechanical logic operations ${ }^{30}$. Although the system demonstrated here is in the classical regime with large mode occupation, where the decoherence is governed by the energy relaxation ${ }^{29}$, these techniques could also be extended to the quantum regime with vibration modes at sufficiently high frequency ${ }^{11}$. This in turn leads to the exciting possibility of quantum-coherent coupling and entanglement between two distinct macroscopic mechanical objects ${ }^{15,16,27}$. 
Pulse sequence

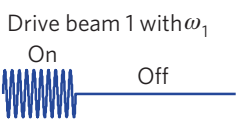

Pump beam 1 with $\omega_{\mathrm{p}}$

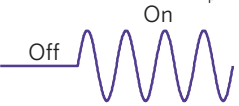

Measure beam 2 at $\omega_{2}$

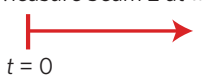

d

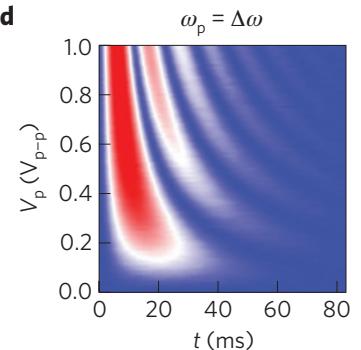

h

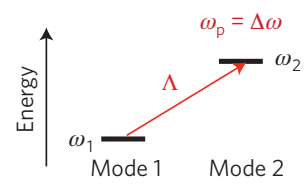

b

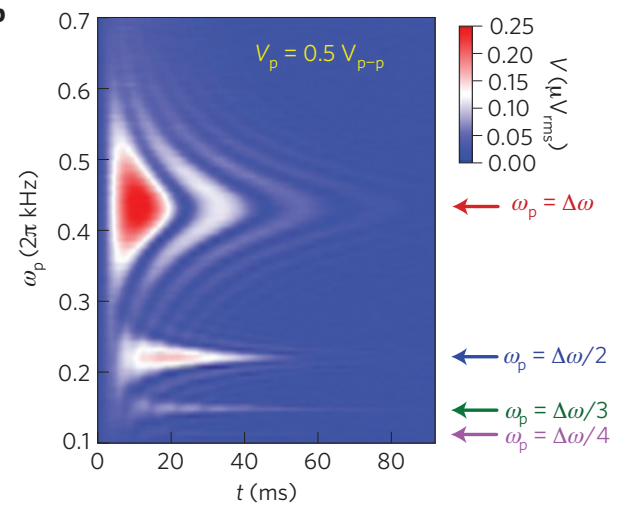

Experiment

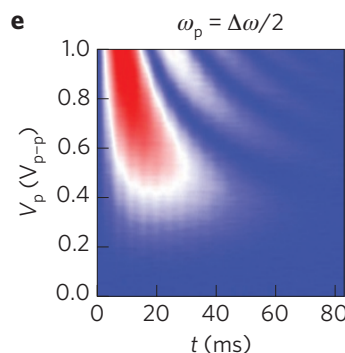

i

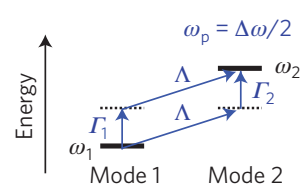

f

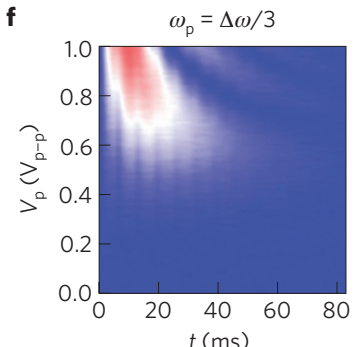

j

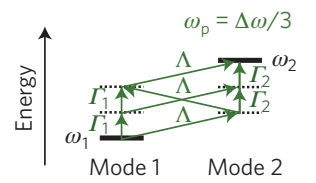

Simulation

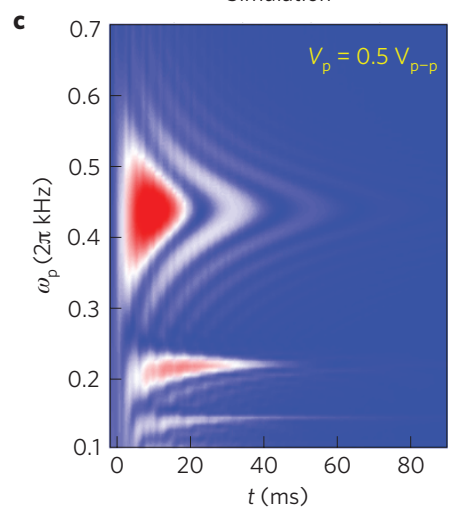

g

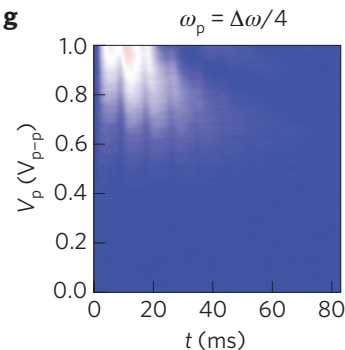

k

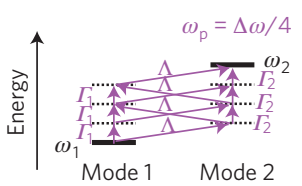

Figure 3 | Coherent energy exchange between the two modes. a, Pulse sequence used for the measurements. The sinusoidal drive pulse voltage $\left(V_{d}=1.5 \mathrm{mV} V_{p-p}\right)$ with $\omega_{d}=\omega_{1}$ was applied to gate $\mathrm{D}$ with a period of $0.1 \mathrm{~s}$. Then, the sinusoidal pump pulse with the frequency, $\omega_{\mathrm{p}}$, was applied to gate $\mathrm{P}$ with the same period. The time response of beam 2 at $\omega_{2}$ was measured through gate M2 with an oscilloscope via a pre-amplifier and a lock-in detector (see Supplementary Information), where the data was averaged 20 times with the oscilloscope. b,c, The $\omega_{p}$ dependence of the time-domain response of

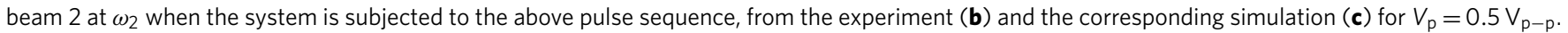
Interference lines with the period of $2 \pi /\left(\omega_{2}-\omega_{1}\right) \simeq 2.3 \mathrm{~ms}$ are found in the calculation (c), which were experimentally eliminated by lock-in filtering (b). $\mathbf{d}-\mathbf{g}$, The $V_{p}$ dependence of the time-domain response of beam 2 at $\omega_{2}$ for $\omega_{p}=\Delta \omega / n$, where $n=1,2,3$ and 4 , respectively. $\mathbf{h}$-k, Schematics of one, two, three, and four-pump phonon absorption processes, where $\Lambda$ is the inter-modal coupling coefficient while $\Gamma_{1}$ and $\Gamma_{2}$ are the intra-modal coupling coefficient for mode 1 and mode 2 , respectively.

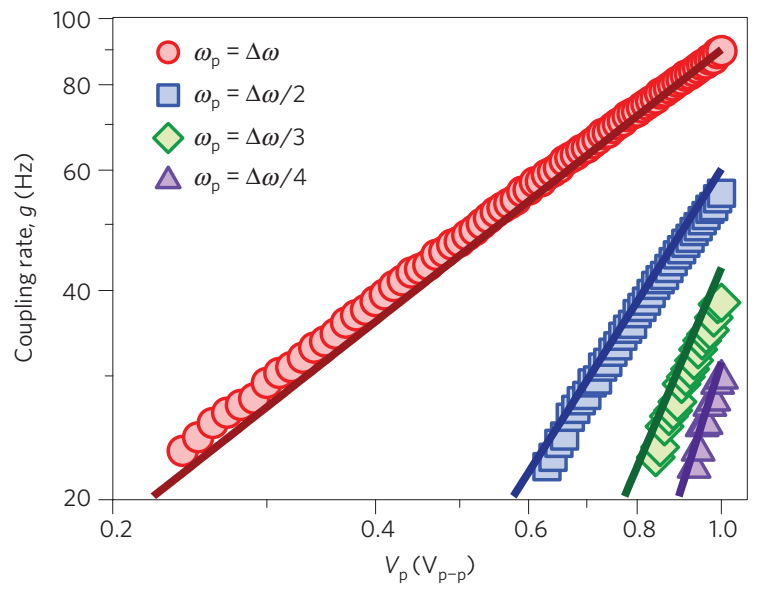

Figure 4 | Coupling rate for the $\boldsymbol{n}$-th order parametric pumping. The logarithmic plots of the coupling rate, $g$, for $\omega_{p}=\Delta \omega / n(n=1,2,3$ and 4$)$ with respect to $V_{p}$, obtained from the Fourier transforms of the time-domain response shown in Fig. 3d-g. Solid lines represent the theoretical values, which are proportional to $V_{p}^{n}$ (see Supplementary Information).

\section{Methods}

The sample was fabricated by photolithography from a heterostructure consisting of 300-nm-thick $\mathrm{Al}_{0.25} \mathrm{Ga}_{0.75} \mathrm{As}, 100$-nm-thick Si-doped $n$-GaAs, 400 -nm-thick undoped $i$-GaAs and 2- $\mu$ m-thick $\mathrm{Al}_{0.65} \mathrm{Ga}_{0.35} \mathrm{As}$ sacrificial layers grown on a GaAs(001) substrate by molecular beam epitaxy. AuGeNi was deposited on the supporting part to obtain an ohmic contact to the conductive $n$-GaAs layer, while 60-nm-thick Au gates were formed on the top of the beams. The suspended structure was completed by a deep mesa and isotropic sacrificial layer etching, where the $40-\mu \mathrm{m}$-separated beams were electrically isolated by the shallow mesa etch. Details of the measurements are described in Supplementary Information.

The simulations were carried out by solving equations (1a) and (1b) with Mathematica 8.0 (Wolfram Research). The derivation of equations (1a) and (1b) and the simulation details are described in Supplementary Information. In the simulation, the only adjustable parameter was the piezoelectric detuning coefficient $\left(\delta \Omega_{1} / \delta V_{\mathrm{p}}=2 \pi \times 0.69 \mathrm{kHz} \mathrm{V}^{-1}\right)$, which determines the relation between $V_{\mathrm{p}}$ and $\Lambda$ $\left(\Gamma_{i}\right)$, and all the remaining parameters were experimentally determined.

Received 27 December 2012; accepted 8 May 2013; published online 2 July 2013; corrected online 4 July 2013

\section{References}

1. Spletzer, M., Raman, A., Wu, A. Q., Xu, X. \& Reifenberger, R. Ultrasensitive mass sensing using mode localization in coupled microcantilevers. Appl. Phys. Lett. 88, 254102 (2006). 
2. Gil-Santos, E. et al. Mass sensing based on deterministic and stochastic resonances of elastically coupled nanocantilevers. Nano Lett. 9, 4122-4127 (2009).

3. Okamoto, H. et al. High-sensitivity charge detection using antisymmetric vibration in coupled micromechanical oscillators. Appl. Phys. Lett. 98, 014103 (2011)

4. Bannon, F. D., Clark, J. R. \& Nguyen, C. T.-C. High-Q HF microelectromechanical filters. IEEE J. Solid-State Circuits 35, 512-526 (2000)

5. Karabalin, R. B. et al. Signal amplification by sensitive control of bifurcation topology. Phys. Rev. Lett. 106, 094102 (2011)

6. Masmanidis, S. C. et al. Multifunctional nanomechanical systems via tunably coupled piezoelectric actuation. Science 317, 780-783 (2007).

7. Shim, S-Bo, Imboden, M. \& Mohanty, P. Synchronized oscillation in coupled nanomechanical oscillators. Science 316, 95-99 (2007).

8. Karabalin, R. B., Cross, M. C. \& Roukes, M. L. Nonlinear dynamics and chaos in two coupled nanomechanical resonators. Phys. Rev. B 79, 165309 (2009).

9. Faust, T. et al. Nonadiabatic dynamics of two strongly coupled nanomechanica resonator modes. Phys. Rev. Lett. 109, 037205 (2012).

10. Okamoto, H., Kamada, T., Onomitsu, K., Mahboob, I. \& Yamaguchi, H. Optical tuning of coupled micromechanical resonators. Appl. Phys. Exp. 2, 062202 (2009).

11. O'Connel, A. D. et al. Quantum ground state and single-phonon control of a mechanical resonator. Nature 464, 697-703 (2010).

12. Verhagen, E., Deléglise, S., Weis, S., Schliesser, A. \& Kippenberg, T. J. Quantum-coherent coupling of a mechanical oscillator to an optical cavity mode. Nature 482, 63-67 (2012).

13. Palomaki, T. A., Harlow, J. W., Teufel, J. D., Simmonds, R. W. \& Lehnert, K. W Coherent state transfer between itinerant microwave fields and a mechanical oscillator. Nature 495, 210-214 (2013)

14. Yamaguchi, H., Okamoto, H. \& Mahboob, I. Coherent control of micro/nanomechanical oscillation using parametric mode mixing. Appl. Phys. Exp. 5, 014001 (2012).

15. Brown, K. R. et al. Coupled quantized mechanical oscillators. Nature 471, 196-199 (2011).

16. Harlander, M., Lechner, R., Brownnutt, M., Blatt, R. \& Hänsel, W. Trapped-ion antennae for the transmission of quantum information. Nature 471, 200-203 (2011).

17. Huygens, C. in Horologium Oscillatorium [The Pendulum Clock] (Apud F. Muguet, 1673).

18. Pikovsky, A., Rosenblum, M. \& Kurths, J. Synchronization: A Universal Concept in Nonlinear Sciences (Cambridge Univ. Press, 2001).

19. Mahboob, I., Nishiguchi, K., Okamoto, H. \& Yamaguchi, H. Phonon-cavity electromechanics. Nature Phys. 8, 387-392 (2012).

20. Yamaguchi, H. \& Mahboob, I. Parametric mode mixing in asymmetric doubly clamped beam resonators. New J. Phys. 15, 015023 (2013).
21. Ding, L. et al. High frequency GaAs nano-optomechanical disk resonator. Phys. Rev. Lett. 105, 263903 (2010).

22. Cole, G. D., Wilson-Rae, I., Werbach, K., Vanner, M. R. \& Aspelmeyer, M. Phonon-tunnelling dissipation in mechanical resonators. Nature Commun. 2 231 (2011)

23. Okamoto, H., Ito, D., Onomitsu, K. \& Yamaguchi, H. Thermoelastic damping in GaAs micromechanical resonators. Phys. Stat. Sol. C 5, 2920-2922 (2008).

24. Weis, S. et al. Optomechanically induced transparency. Science 330, 1520-1523 (2010).

25. Safavi-Naeini, A. H. et al. Electromagnetically induced transparency and slow light with optomechanics. Nature 472, 69-73 (2011).

26. Teufel, J. et al. Circuit cavity electromechanics in the strong-coupling regime. Nature 471, 204-208 (2011).

27. Ansmann, M. et al. Violation of Bell's inequality in Josephson phase qubits. Nature 461, 504-506 (2009).

28. Kippenberg, T. J., Holzwarth, R. \& Diddams, S. A. Microresonator-based optical frequency combs. Science 332, 555-559 (2011).

29. Faust, T., Rieger, J., Seitner, M. J., Kotthaus, J. P. \& Weig, E. M. Coherent control of a classical nanomechanical two-level system. Nature Phys. http://dx.doi.org/10.1038/nphys2666 (2013).

30. Mahboob, I., Flurin, E., Nishiguchi, K., Fujiwara, A. \& Yamaguchi, H. Interconnect-free parallel logic circuits in a single mechanical resonator. Nature Commun. 2, 198 (2011).

\section{Acknowledgements}

H.O. thanks A. Taspinar for supporting the data analysis. The authors acknowledge T. Faust, J. P. Kotthaus and E. M. Weig for their critical reading of the manuscript. This work was partly supported by JSPS KAKENHI (23241046 \& 20246064)

\section{Author contributions}

H.O. designed and fabricated the sample with support from K.O., C-Y.C. and E.Y.C. The measurements and data analysis were performed by H.O. and A.G. The simulation was performed by H.Y. The paper was written by H.O., based on the discussions with I.M. and H.Y. The project was planned by H.Y.

\section{Additional information}

Supplementary information is available in the online version of the paper. Reprints and permissions information is available online at www.nature.com/reprints. Correspondence and requests for materials should be addressed to H.O.

\section{Competing financial interests}

The authors declare no competing financial interests. 


\section{ERRATUM}

\section{Coherent phonon manipulation in coupled mechanical resonators}

Hajime Okamoto, Adrien Gourgout, Chia-Yuan Chang, Koji Onomitsu, Imran Mahboob, Edward Yi Chang and Hiroshi Yamaguchi

Nature Physics 9, 480-484 (2013); published online 2 July 2013; corrected after print 19 August 2013.

In the version of this Letter originally published, the second author affiliation should have read "The Department of Materials Science and Engineering, National Chiao-Tung University, Hsinchu 980-8578, Taiwan”. This error has now been corrected in the HTML and PDF versions of the Letter. 